\title{
Measurement of $\sigma(p \bar{p} \rightarrow Z) \cdot \operatorname{Br}(Z \rightarrow \tau \tau)$ at $\sqrt{s}=1.96 \mathrm{TeV}$
}

V.M. Abazov, ${ }^{34}$ B. Abbott, ${ }^{71}$ M. Abolins, ${ }^{62}$ B.S. Acharya, ${ }^{28}$ M. Adams,${ }^{49}$ T. Adams, ${ }^{47}$ M. Agelou, ${ }^{17}$ J.-L. Agram, ${ }^{18}$ S.H. Ahn, ${ }^{30}$ M. Ahsan,${ }^{56}$ G.D. Alexeev,${ }^{34}$ G. Alkhazov, ${ }^{38}$ A. Alton, ${ }^{61}$ G. Alverson, ${ }^{60}$ G.A. Alves,${ }^{2}$ M. Anastasoaie,${ }^{33}$

T. Andeen, ${ }^{51}$ S. Anderson, ${ }^{43}$ B. Andrieu, ${ }^{16}$ Y. Arnoud, ${ }^{13}$ A. Askew,${ }^{75}$ B. Åsman,,${ }^{39}$ O. Atramentov,${ }^{54}$

C. Autermann, ${ }^{20}$ C. Avila,${ }^{7}$ F. Badaud,${ }^{12}$ A. Baden, ${ }^{58}$ B. Baldin, ${ }^{48}$ P.W. Balm,${ }^{32}$ S. Banerjee,${ }^{28}$ E. Barberis,${ }^{60}$

P. Bargassa,${ }^{75}$ P. Baringer,${ }^{55}$ C. Barnes,${ }^{41}$ J. Barreto, ${ }^{2}$ J.F. Bartlett,${ }^{48}$ U. Bassler, ${ }^{16}$ D. Bauer,${ }^{52}$ A. Bean,${ }^{55}$

S. Beauceron, ${ }^{16}$ M. Begel,${ }^{67}$ A. Bellavance,${ }^{64}$ S.B. Beri, ${ }^{26}$ G. Bernardi,${ }^{16}$ R. Bernhard,${ }^{48, *}$ I. Bertram, ${ }^{40}$

M. Besançon, ${ }^{17}$ R. Beuselinck ${ }^{41}$ V.A. Bezzubov,${ }^{37}$ P.C. Bhat,${ }^{48}$ V. Bhatnagar, ${ }^{26}$ M. Binder,${ }^{24}$ C. Biscarat, ${ }^{40}$

K.M. Black, ${ }^{59}$ I. Blackler, ${ }^{41}$ G. Blazey,${ }^{50}$ F. Blekman, ${ }^{32}$ S. Blessing, ${ }^{47}$ D. Bloch,${ }^{18}$ U. Blumenschein,${ }^{22}$

A. Boehnlein, ${ }^{48}$ O. Boeriu ${ }^{53}$ T.A. Bolton,${ }^{56}$ F. Borcherding, ${ }^{48}$ G. Borissov,${ }^{40} \mathrm{~K}$. Bos,${ }^{32}$ T. Bose,${ }^{66}$ A. Brandt,${ }^{73}$

R. Brock,${ }^{62}$ G. Brooijmans, ${ }^{66}$ A. Bross,${ }^{48}$ N.J. Buchanan,${ }^{47}$ D. Buchholz,${ }^{51}$ M. Buehler,${ }^{49}$ V. Buescher, ${ }^{22}$

S. Burdin, ${ }^{48}$ T.H. Burnett, ${ }^{77}$ E. Busato,${ }^{16}$ J.M. Butler, ${ }^{59}$ J. Bystricky,${ }^{17}$ W. Carvalho, ${ }^{3}$ B.C.K. Casey,${ }^{72}$

N.M. Cason, ${ }^{53}$ H. Castilla-Valdez, ${ }^{31}$ S. Chakrabarti, ${ }^{28}$ D. Chakraborty,${ }^{50}$ K.M. Chan, ${ }^{67}$ A. Chandra, ${ }^{28}$ D. Chapin, ${ }^{72}$ F. Charles, ${ }^{18}$ E. Cheu, ${ }^{43}$ L. Chevalier, ${ }^{17}$ D.K. Cho, ${ }^{67}$ S. Choi,${ }^{46}$ B. Choudhary,${ }^{27}$ T. Christiansen, ${ }^{24}$ L. Christofek, ${ }^{55}$ D. Claes,${ }^{64}$ B. Clément, ${ }^{18}$ C. Clément,${ }^{39}$ Y. Coadou,${ }^{5}$ M. Cooke,${ }^{75}$ W.E. Cooper, ${ }^{48}$ D. Coppage,${ }^{55}$ M. Corcoran,${ }^{75}$

A. Cothenet, ${ }^{14}$ M.-C. Cousinou, ${ }^{14}$ B. Cox,${ }^{42}$ S. Crépé-Renaudin,${ }^{13}$ M. Cristetiu, ${ }^{46}$ D. Cutts,${ }^{72}$ H. da Motta, ${ }^{2}$

B. Davies ${ }^{40}$ G. Davies ${ }^{41}$ G.A. Davis,${ }^{51}$ K. De,${ }^{73}$ P. de Jong, ${ }^{32}$ S.J. de Jong, ${ }^{33}$ E. De La Cruz-Burelo, ${ }^{31}$

C. De Oliveira Martins,${ }^{3}$ S. Dean,${ }^{42}$ F. Déliot,${ }^{17}$ M. Demarteau, ${ }^{48}$ R. Demina,${ }^{67}$ P. Demine, ${ }^{17}$ D. Denisov ${ }^{48}$

S.P. Denisov, ${ }^{37}$ S. Desai, ${ }^{68}$ H.T. Diehl, ${ }^{48}$ M. Diesburg, ${ }^{48}$ M. Doidge,${ }^{40}$ H. Dong, ${ }^{68}$ S. Doulas,${ }^{60}$ L.V. Dudko, ${ }^{36}$

L. Duflot, ${ }^{15}$ S.R. Dugad ${ }^{28}$ A. Duperrin, ${ }^{14}$ J. Dyer ${ }^{62}$ A. Dyshkant,${ }^{50}$ M. Eads,${ }^{50}$ D. Edmunds, ${ }^{62}$ T. Edwards, ${ }^{42}$

J. Ellison, ${ }^{46}$ J. Elmsheuser, ${ }^{24}$ J.T. Eltzroth ${ }^{73}$ V.D. Elvira, ${ }^{48}$ S. Eno, ${ }^{58}$ P. Ermolov, ${ }^{36}$ O.V. Eroshin, ${ }^{37}$ J. Estrada, ${ }^{48}$

D. Evans, ${ }^{41}$ H. Evans,${ }^{66}$ A. Evdokimov, ${ }^{35}$ V.N. Evdokimov, ${ }^{37}$ J. Fast, ${ }^{48}$ S.N. Fatakia, ${ }^{59}$ L. Feligioni, ${ }^{59}$

T. Ferbel,${ }^{67}$ F. Fiedler ${ }^{24}$ F. Filthaut, ${ }^{33}$ W. Fisher, ${ }^{65}$ H.E. Fisk,${ }^{48}$ M. Fortner, ${ }^{50}$ H. Fox,${ }^{22}$ W. Freeman, ${ }^{48}$

S. Fu, ${ }^{48}$ S. Fuess, ${ }^{48}$ T. Gadfort, ${ }^{77}$ C.F. Galea ${ }^{33}$ E. Gallas ${ }^{48}$ E. Galyaev,${ }^{53}$ C. Garcia, ${ }^{67}$ A. Garcia-Bellido, ${ }^{77}$

J. Gardner, ${ }^{55}$ V. Gavrilov, ${ }^{35}$ P. Gay ${ }^{12}$ D. Gelé, ${ }^{18}$ R. Gelhaus, ${ }^{46}$ K. Genser ${ }^{48}$ C.E. Gerber ${ }^{49}$ Y. Gershtein, ${ }^{72}$ G. Ginther, ${ }^{67}$ T. Golling, ${ }^{21}$ B. Gómez,${ }^{7}$ K. Gounder, ${ }^{48}$ A. Goussiou, ${ }^{53}$ P.D. Grannis,${ }^{68}$ S. Greder, ${ }^{18}$ H. Greenlee,${ }^{48}$ Z.D. Greenwood, ${ }^{57}$ E.M. Gregores, ${ }^{4}$ Ph. Gris,,${ }^{12}$ J.-F. Grivaz, ${ }^{15}$ L. Groer, ${ }^{66}$ S. Grünendahl,,${ }^{48}$ M.W. Grünewald ${ }^{29}$ S.N. Gurzhiev ${ }^{37}$ G. Gutierrez ${ }^{48}$ P. Gutierrez, ${ }^{71}$ A. Haas, ${ }^{66}$ N.J. Hadley, ${ }^{58}$ S. Hagopian,,${ }^{47}$ I. Hall, ${ }^{71}$ R.E. Hall, ${ }^{45}$

C. Han, ${ }^{61}$ L. Han, ${ }^{42}$ K. Hanagaki, ${ }^{48}$ K. Harder, ${ }^{56}$ R. Harrington, ${ }^{60}$ J.M. Hauptman,${ }^{54}$ R. Hauser,${ }^{62}$ J. Hays, ${ }^{51}$

T. Hebbeker, ${ }^{20}$ D. Hedin, ${ }^{50}$ J.M. Heinmiller ${ }^{49}$ A.P. Heinson, ${ }^{46}$ U. Heintz, ${ }^{59}$ C. Hensel,${ }^{55}$ G. Hesketh, ${ }^{60}$

M.D. Hildreth, ${ }^{53}$ R. Hirosky, ${ }^{76}$ J.D. Hobbs,${ }^{68}$ B. Hoeneisen, ${ }^{11}$ M. Hohlfeld, ${ }^{23}$ S.J. Hong,${ }^{30}$ R. Hooper,${ }^{72}$ P. Houben, ${ }^{32}$

Y. Hu, ${ }^{68}$ J. Huang, ${ }^{52}$ I. Iashvili, ${ }^{46}$ R. Illingworth,${ }^{48}$ A.S. Ito, ${ }^{48}$ S. Jabeen, ${ }^{55}$ M. Jaffré, ${ }^{15}$ S. Jain, ${ }^{71}$ V. Jain, ${ }^{69}$

K. Jakobs, ${ }^{22}$ A. Jenkins, ${ }^{41}$ R. Jesik, ${ }^{41}$ K. Johns, ${ }^{43}$ M. Johnson, ${ }^{48}$ A. Jonckheere ${ }^{48}$ P. Jonsson, ${ }^{41}$ H. Jöstlein, ${ }^{48}$

A. Juste ${ }^{48}$ D. Käfer, ${ }^{20}$ W. Kahl,${ }^{56}$ S. Kahn, ${ }^{69}$ E. Kajfasz,${ }^{14}$ A.M. Kalinin, ${ }^{34}$ J. Kalk, ${ }^{62}$ D. Karmanov,${ }^{36}$

J. Kasper, ${ }^{59}$ D. Kau ${ }^{47}$ R. Kaur, ${ }^{26}$ R. Kehoe,${ }^{74}$ S. Kermiche, ${ }^{14}$ S. Kesisoglou,${ }^{72}$ A. Khanov, ${ }^{67}$ A. Kharchilava, ${ }^{53}$

Y.M. Kharzheev ${ }^{34}$ K.H. Kim,${ }^{30}$ B. Klima ${ }^{48}$ M. Klute, ${ }^{21}$ J.M. Kohli, ${ }^{26}$ M. Kopal, ${ }^{71}$ V.M. Korablev ${ }^{37}$ J. Kotcher ${ }^{69}$

B. Kothari, ${ }^{66}$ A. Koubarovsky, ${ }^{36}$ A.V. Kozelov, ${ }^{37}$ J. Kozminski, ${ }^{62}$ S. Krzywdzinski, ${ }^{48}$ S. Kuleshov, ${ }^{35}$ Y. Kulik, ${ }^{48}$

A. Kumar, ${ }^{27}$ S. Kunori, ${ }^{58}$ A. Kupco,${ }^{10}$ T. Kurča, ${ }^{19}$ S. Lager, ${ }^{39}$ N. Lahrichi, ${ }^{17}$ G. Landsberg, ${ }^{72}$ J. Lazoflores,${ }^{47}$

A.-C. Le Bihan, ${ }^{18}$ P. Lebrun, ${ }^{19}$ S.W. Lee ${ }^{30}$ W.M. Lee ${ }^{47}$ A. Leflat,${ }^{36}$ F. Lehner,${ }^{48, *}$ C. Leonidopoulos,${ }^{66}$ P. Lewis ${ }^{41}$

J. Li, ${ }^{73}$ Q.Z. Li ${ }^{48}$ J.G.R. Lima, ${ }^{50}$ D. Lincoln,${ }^{48}$ S.L. Linn,${ }^{47}$ J. Linnemann, ${ }^{62}$ V.V. Lipaev ${ }^{37}$ R. Lipton, ${ }^{48}$

L. Lobo, ${ }^{41}$ A. Lobodenko, ${ }^{38}$ M. Lokajicek, ${ }^{10}$ A. Lounis, ${ }^{18}$ H.J. Lubatti, ${ }^{77}$ L. Lueking,${ }^{48}$ M. Lynker, ${ }^{53}$ A.L. Lyon, ${ }^{48}$

A.K.A. Maciel, ${ }^{50}$ R.J. Madaras, ${ }^{44}$ P. Mättig, ${ }^{25}$ A. Magerkurth, ${ }^{61}$ A.-M. Magnan, ${ }^{13}$ N. Makovec, ${ }^{15}$ P.K. Mal, ${ }^{28}$

S. Malik, ${ }^{57}$ V.L. Malyshev, ${ }^{34}$ H.S. Mao, ${ }^{6}$ Y. Maravin, ${ }^{48}$ M. Martens, ${ }^{48}$ S.E.K. Mattingly, ${ }^{72}$ A.A. Mayorov ${ }^{37}$

R. McCarthy, ${ }^{68}$ R. McCroskey ${ }^{43}$ D. Meder, ${ }^{23}$ H.L. Melanson, ${ }^{48}$ A. Melnitchouk, ${ }^{63}$ A. Mendes,${ }^{14}$ M. Merkin, ${ }^{36}$

K.W. Merritt ${ }^{48}$ A. Meyer, ${ }^{20}$ M. Michaut, ${ }^{17}$ H. Miettinen, ${ }^{75}$ J. Mitrevski, ${ }^{66}$ N. Mokhov, ${ }^{48}$ J. Molina,${ }^{3}$

N.K. Mondal, ${ }^{28}$ R.W. Moore, ${ }^{5}$ G.S. Muanza, ${ }^{19}$ M. Mulders, ${ }^{48}$ Y.D. Mutaf, ${ }^{68}$ E. Nagy, ${ }^{14}$ M. Narain, ${ }^{59}$

N.A. Naumann, ${ }^{33}$ H.A. Neal, ${ }^{61}$ J.P. Negret,${ }^{7}$ S. Nelson, ${ }^{47}$ P. Neustroev, ${ }^{38}$ C. Noeding, ${ }^{22}$ A. Nomerotski, ${ }^{48}$

S.F. Novaes, ${ }^{4}$ T. Nunnemann, ${ }^{24}$ E. Nurse,${ }^{42}$ V. O’Dell, ${ }^{48}$ D.C. O’Neil,${ }^{5}$ V. Oguri, ${ }^{3}$ N. Oliveira, ${ }^{3}$ N. Oshima, ${ }^{48}$ G.J. Otero y Garzón, ${ }^{49}$ P. Padley, ${ }^{75}$ N. Parashar, ${ }^{57}$ J. Park, ${ }^{30}$ S.K. Park, ${ }^{30}$ J. Parsons, ${ }^{66}$ R. Partridge,${ }^{72}$ N. Parua, ${ }^{68}$ 
A. Patwa ${ }^{69}$ P.M. Perea,${ }^{46}$ E. Perez,${ }^{17}$ P. Pétroff,${ }^{15}$ M. Petteni, ${ }^{41}$ L. Phaf,${ }^{32}$ R. Piegaia, ${ }^{1}$ M.-A. Pleier,${ }^{67}$ P.L.M. Podesta-Lerma, ${ }^{31}$ V.M. Podstavkov, ${ }^{48}$ Y. Pogorelov, ${ }^{53}$ B.G. Pope, ${ }^{62}$ W.L. Prado da Silva, ${ }^{3}$ H.B. Prosper, ${ }^{47}$ S. Protopopescu, ${ }^{69}$ J. Qian, ${ }^{61}$ A. Quadt, ${ }^{21}$ B. Quinn, ${ }^{63}$ K.J. Rani ${ }^{28}$ K. Ranjan, ${ }^{27}$ P.A. Rapidis, ${ }^{48}$ P.N. Ratoff, ${ }^{40}$ N.W. Reay ${ }^{56}$ S. Reucroft,${ }^{60}$ M. Rijssenbeek,${ }^{68}$ I. Ripp-Baudot,${ }^{18}$ F. Rizatdinova,${ }^{56}$ C. Royon, ${ }^{17}$ P. Rubinov,${ }^{48}$

R. Ruchti, ${ }^{53}$ V.I. Rud ${ }^{36}$ G. Sajot, ${ }^{13}$ A. Sánchez-Hernández,${ }^{31}$ M.P. Sanders, ${ }^{42}$ A. Santoro, ${ }^{3}$ G. Savage, ${ }^{48}$

L. Sawyer, ${ }^{57}$ T. Scanlon, ${ }^{41}$ D. Schaile, ${ }^{24}$ R.D. Schamberger,${ }^{68}$ H. Schellman, ${ }^{51}$ P. Schieferdecker, ${ }^{24}$ C. Schmitt, ${ }^{25}$ A.A. Schukin, ${ }^{37}$ A. Schwartzman, ${ }^{65}$ R. Schwienhorst, ${ }^{62}$ S. Sengupta, ${ }^{47}$ H. Severini, ${ }^{71}$ E. Shabalina, ${ }^{49}$ M. Shamim, ${ }^{56}$

V. Shary, ${ }^{17}$ W.D. Shephard, ${ }^{53}$ R.K. Shivpuri, ${ }^{27}$ D. Shpakov ${ }^{60}$ R.A. Sidwell,${ }^{56}$ V. Simak,${ }^{9}$ V. Sirotenko, ${ }^{48}$

P. Skubic, ${ }^{71}$ P. Slattery, ${ }^{67}$ R.P. Smith, ${ }^{48}$ K. Smolek, ${ }^{9}$ G.R. Snow,${ }^{64}$ J. Snow,${ }^{70}$ S. Snyder ${ }^{69}$ S. Söldner-Rembold, ${ }^{42}$

X. Song, ${ }^{50}$ Y. Song, ${ }^{73}$ L. Sonnenschein, ${ }^{59}$ A. Sopczak ${ }^{40}$ M. Sosebee ${ }^{73}$ K. Soustruznik, ${ }^{8}$ M. Souza,${ }^{2}$

B. Spurlock ${ }^{73}$ N.R. Stanton, ${ }^{56}$ J. Stark, ${ }^{13}$ J. Steele,${ }^{57}$ G. Steinbrück, ${ }^{66}$ K. Stevenson, ${ }^{52}$ V. Stolin,${ }^{35}$ A. Stone,${ }^{49}$

D.A. Stoyanova, ${ }^{37}$ J. Strandberg, ${ }^{39}$ M.A. Strang, ${ }^{73}$ M. Strauss, ${ }^{71}$ R. Ströhmer, ${ }^{24}$ D. Strom, ${ }^{51}$ M. Strovink, ${ }^{44}$

L. Stutte, ${ }^{48}$ S. Sumowidagdo, ${ }^{47}$ A. Sznajder,${ }^{3}$ M. Talby, ${ }^{14}$ P. Tamburello, ${ }^{43}$ W. Taylor, ${ }^{5}$ P. Telford, ${ }^{42}$ J. Temple, ${ }^{43}$

E. Thomas, ${ }^{14}$ B. Thooris,${ }^{17}$ M. Tomoto, ${ }^{48}$ T. Toole, ${ }^{58}$ J. Torborg, ${ }^{53}$ S. Towers, ${ }^{68}$ T. Trefzger,${ }^{23}$ S. Trincaz-Duvoid, ${ }^{16}$

B. Tuchming, ${ }^{17}$ C. Tully, ${ }^{65}$ A.S. Turcot,${ }^{69}$ P.M. Tuts,${ }^{66}$ L. Uvarov ${ }^{38}$ S. Uvarov, ${ }^{38}$ S. Uzunyan, ${ }^{50}$ B. Vachon, ${ }^{5}$

R. Van Kooten, ${ }^{52}$ W.M. van Leeuwen, ${ }^{32}$ N. Varelas, ${ }^{49}$ E.W. Varnes,${ }^{43}$ I.A. Vasilyev, ${ }^{37}$ M. Vaupel,${ }^{25}$ P. Verdier ${ }^{15}$

L.S. Vertogradov, ${ }^{34}$ M. Verzocchi, ${ }^{58}$ F. Villeneuve-Seguier, ${ }^{41}$ J.-R. Vlimant,${ }^{16}$ E. Von Toerne,${ }^{56}$ M. Vreeswijk, ${ }^{32}$

T. Vu Anh, ${ }^{15}$ H.D. Wahl, ${ }^{47}$ R. Walker ${ }^{41}$ L. Wang, ${ }^{58}$ Z.-M. Wang, ${ }^{68}$ J. Warchol, ${ }^{53}$ M. Warsinsky, ${ }^{21}$

G. Watts, ${ }^{77}$ M. Wayne, ${ }^{53}$ M. Weber,${ }^{48}$ H. Weerts, ${ }^{62}$ M. Wegner,${ }^{20}$ N. Wermes,${ }^{21}$ A. White,${ }^{73}$ V. White,${ }^{48}$

D. Whiteson, ${ }^{44}$ D. Wicke, ${ }^{48}$ D.A. Wijngaarden, ${ }^{33}$ G.W. Wilson, ${ }^{55}$ S.J. Wimpenny, ${ }^{46}$ J. Wittlin, ${ }^{59}$ M. Wobisch, ${ }^{48}$

J. Womersley, ${ }^{48}$ D.R. Wood, ${ }^{60}$ T.R. Wyatt, ${ }^{42}$ Q. Xu ${ }^{61}$ N. Xuan,${ }^{53}$ S. Yacoob, ${ }^{51}$ R. Yamada, ${ }^{48}$ M. Yan, ${ }^{58}$

T. Yasuda ${ }^{48}$ Y.A. Yatsunenko, ${ }^{34}$ Y. Yen,${ }^{25}$ K. Yip,${ }^{69}$ S.W. Youn, ${ }^{51}$ J. Yu, ${ }^{73}$ A. Yurkewicz,${ }^{68}$ A. Zabi, ${ }^{15}$

A. Zatserklyaniy, ${ }^{50}$ M. Zdrazil,${ }^{68}$ C. Zeitnitz, ${ }^{23}$ D. Zhang, ${ }^{48}$ X. Zhang, ${ }^{71}$ T. Zhao, ${ }^{77}$ Z. Zhao, ${ }^{61}$ B. Zhou,${ }^{61}$ J. Zhu, ${ }^{58}$

M. Zielinski, ${ }^{67}$ D. Zieminska, ${ }^{52}$ A. Zieminski, ${ }^{52}$ R. Zitoun, ${ }^{68}$ V. Zutshi, ${ }^{50}$ E.G. Zverev ${ }^{36}$ and A. Zylberstejn ${ }^{17}$ (DØ Collaboration)

${ }^{1}$ Universidad de Buenos Aires, Buenos Aires, Argentina

${ }^{2}$ LAFEX, Centro Brasileiro de Pesquisas Físicas, Rio de Janeiro, Brazil

${ }^{3}$ Universidade do Estado do Rio de Janeiro, Rio de Janeiro, Brazil

${ }^{4}$ Instituto de Física Teórica, Universidade Estadual Paulista, São Paulo, Brazil

${ }^{5}$ University of Alberta, Edmonton, Alberta, Canada, Simon Fraser University, Burnaby, British Columbia, Canada,

York University, Toronto, Ontario, Canada, and McGill University, Montreal, Quebec, Canada

${ }^{6}$ Institute of High Energy Physics, Beijing, People's Republic of China

${ }^{7}$ Universidad de los Andes, Bogotá, Colombia

${ }^{8}$ Center for Particle Physics, Charles University, Prague, Czech Republic

${ }^{9}$ Czech Technical University, Prague, Czech Republic

${ }^{10}$ Institute of Physics, Academy of Sciences, Center for Particle Physics, Prague, Czech Republic

${ }^{11}$ Universidad San Francisco de Quito, Quito, Ecuador

${ }^{12}$ Laboratoire de Physique Corpusculaire, IN2P3-CNRS, Université Blaise Pascal, Clermont-Ferrand, France

${ }^{13}$ Laboratoire de Physique Subatomique et de Cosmologie, IN2P3-CNRS, Universite de Grenoble 1, Grenoble, France

${ }^{14}$ CPPM, IN2P3-CNRS, Université de la Méditerranée, Marseille, France

${ }^{15}$ Laboratoire de l'Accélérateur Linéaire, IN2P3-CNRS, Orsay, France

${ }^{16}$ LPNHE, IN2P3-CNRS, Universités Paris VI and VII, Paris, France

${ }^{17}$ DAPNIA/Service de Physique des Particules, CEA, Saclay, France

${ }^{18}$ IReS, IN2P3-CNRS, Université Louis Pasteur, Strasbourg, France, and Université de Haute Alsace, Mulhouse, France

${ }^{19}$ Institut de Physique Nucléaire de Lyon, IN2P3-CNRS, Université Claude Bernard, Villeurbanne, France

${ }^{20}$ III. Physikalisches Institut A, RWTH Aachen, Aachen, Germany

${ }^{21}$ Physikalisches Institut, Universität Bonn, Bonn, Germany

${ }^{22}$ Physikalisches Institut, Universität Freiburg, Freiburg, Germany

${ }^{23}$ Institut für Physik, Universität Mainz, Mainz, Germany

${ }^{24}$ Ludwig-Maximilians-Universität München, München, Germany

${ }^{25}$ Fachbereich Physik, University of Wuppertal, Wuppertal, Germany

${ }^{26}$ Panjab University, Chandigarh, India

${ }^{27}$ Delhi University, Delhi, India

${ }^{28}$ Tata Institute of Fundamental Research, Mumbai, India

${ }^{29}$ University College Dublin, Dublin, Ireland

${ }^{30}$ Korea Detector Laboratory, Korea University, Seoul, Korea

${ }^{31}$ CINVESTAV, Mexico City, Mexico

${ }^{32}$ FOM-Institute NIKHEF and University of Amsterdam/NIKHEF, Amsterdam, The Netherlands 
${ }^{33}$ University of Nijmegen/NIKHEF, Nijmegen, The Netherlands

${ }^{34}$ Joint Institute for Nuclear Research, Dubna, Russia

${ }^{35}$ Institute for Theoretical and Experimental Physics, Moscow, Russia

${ }^{36}$ Moscow State University, Moscow, Russia

${ }^{37}$ Institute for High Energy Physics, Protvino, Russia

${ }^{38}$ Petersburg Nuclear Physics Institute, St. Petersburg, Russia

${ }^{39}$ Lund University, Lund, Sweden, Royal Institute of Technology and Stockholm University, Stockholm, Sweden, and Uppsala University, Uppsala, Sweden

${ }^{40}$ Lancaster University, Lancaster, United Kingdom

${ }^{41}$ Imperial College, London, United Kingdom

${ }^{42}$ University of Manchester, Manchester, United Kingdom

${ }^{43}$ University of Arizona, Tucson, Arizona 85721, USA

${ }^{44}$ Lawrence Berkeley National Laboratory and University of California, Berkeley, California 94720, USA

${ }^{45}$ California State University, Fresno, California 93740, USA

${ }^{46}$ University of California, Riverside, California 92521, USA

${ }^{47}$ Florida State University, Tallahassee, Florida 32306, USA

${ }^{48}$ Fermi National Accelerator Laboratory, Batavia, Illinois 60510, USA

${ }^{49}$ University of Illinois at Chicago, Chicago, Illinois 60607, USA

${ }^{50}$ Northern Illinois University, DeKalb, Illinois 60115, USA

${ }^{51}$ Northwestern University, Evanston, Illinois 60208, USA

${ }^{52}$ Indiana University, Bloomington, Indiana 47405, USA

${ }^{53}$ University of Notre Dame, Notre Dame, Indiana 46556, USA

${ }^{54}$ Iowa State University, Ames, Iowa 50011, USA

${ }^{55}$ University of Kansas, Lawrence, Kansas 66045, USA

${ }^{56}$ Kansas State University, Manhattan, Kansas 66506, USA

${ }^{57}$ Louisiana Tech University, Ruston, Louisiana 71272, USA

${ }^{58}$ University of Maryland, College Park, Maryland 20742, USA

${ }^{59}$ Boston University, Boston, Massachusetts 02215, USA

${ }^{60}$ Northeastern University, Boston, Massachusetts 02115, USA

${ }^{61}$ University of Michigan, Ann Arbor, Michigan 48109, USA

${ }^{62}$ Michigan State University, East Lansing, Michigan 48824, USA

${ }^{63}$ University of Mississippi, University, Mississippi 38677, USA

${ }^{64}$ University of Nebraska, Lincoln, Nebraska 68588, USA

${ }^{65}$ Princeton University, Princeton, New Jersey 08544, USA

${ }^{66}$ Columbia University, New York, New York 10027, USA

${ }^{67}$ University of Rochester, Rochester, New York 14627, USA

${ }^{68}$ State University of New York, Stony Brook, New York 11794, USA

${ }^{69}$ Brookhaven National Laboratory, Upton, New York 11973, USA

${ }^{70}$ Langston University, Langston, Oklahoma 73050, USA

${ }^{71}$ University of Oklahoma, Norman, Oklahoma 73019, USA

${ }^{72}$ Brown University, Providence, Rhode Island 02912, USA

${ }^{73}$ University of Texas, Arlington, Texas 76019, USA

${ }^{74}$ Southern Methodist University, Dallas, Texas 75275, USA

${ }^{75}$ Rice University, Houston, Texas 77005, USA

${ }^{76}$ University of Virginia, Charlottesville, Virginia 22901, USA

${ }^{77}$ University of Washington, Seattle, Washington 98195, USA

(Dated: November 5, 2018)

We present a measurement of the cross section for $Z$ production times the branching fraction to $\tau$ leptons, $\sigma \cdot \operatorname{Br}\left(Z \rightarrow \tau^{+} \tau^{-}\right)$, in $p \bar{p}$ collisions at $\sqrt{s}=1.96 \mathrm{TeV}$ in the channel in which one $\tau$ decays into $\mu \nu_{\mu} \nu_{\tau}$, and the other into hadrons $+\nu_{\tau}$ or $e \nu_{e} \nu_{\tau}$. The data sample corresponds to an integrated luminosity of $226 \mathrm{pb}^{-1}$ collected with the $\mathrm{D} \varnothing$ detector at the Fermilab Tevatron collider. The final sample contains 2008 candidate events with an estimated background of $55 \%$. From this we obtain $\sigma \cdot \operatorname{Br}\left(Z \rightarrow \tau^{+} \tau^{-}\right)=237 \pm 15$ (stat) \pm 18 (sys) \pm 15 (lum) pb, in agreement with the standard model prediction.

PACS numbers: 13.38.Dg,13.85.Qk,14.70.Hp

Measurements of the $Z$ boson production cross section times the leptonic branching fraction $(\sigma \cdot \mathrm{Br})$ in $p \bar{p}$ collisions can be used to test standard model (SM) predictions. The $\sigma \cdot \operatorname{Br}$ to $e^{+} e^{-}$and $\mu^{+} \mu^{-}$in $p \bar{p}$ collisions has been measured by the UA1 and UA2 collaborations at $\sqrt{s}=630 \mathrm{GeV}[1]$, by the CDF collaboration at $\sqrt{s}=1.8$ $\mathrm{TeV}$ and $\sqrt{s}=1.96 \mathrm{TeV}[2]$, and by the $\mathrm{D} \varnothing$ collaboration at $\sqrt{s}=1.8 \mathrm{TeV}[\underline{3}]$. The $Z$ boson branching ratio 
to $\tau^{+} \tau^{-}$has been measured with high precision by the CERN $e^{+} e^{-}$collider (LEP) experiments 4]. These measurements are in good agreement with SM expectations and lepton universality. We report here the first measurement of $\sigma \cdot \operatorname{Br}\left(Z \rightarrow \tau^{+} \tau^{-}\right)$in $p \bar{p}$ collisions. This measurement provides a test of the SM as a deviation from the expected value would be an indication of anomalous production of $\tau^{+} \tau^{-}$pairs in $p \bar{p}$ collisions. It also verifies that the $\mathrm{D} \varnothing$ detector can identify isolated $\tau$ leptons in the energy range covered by $Z$ boson decays, which could be critical in the search for non-SM signals such as supersymmetric (SUSY) particles in certain regions of the SUSY parameter space, or heavy resonances decaying into fermion pairs with enhanced coupling to the third generation.

The DØ Run II detector is fully described in [5]; a more succinct description of details relevant to this measurement can be found in [6]. The $Z \rightarrow \tau\left(\rightarrow \mu \nu_{\mu} \nu_{\tau}\right) \tau$ candidate selection strategy focused on one $\tau$ lepton decaying to muon by triggering on the single muon using a three-level triggering system. The first level used the timing and position information in the muon scintillator system to find muon candidates. The second level used digital signal processors to form segments defined in the muon drift chambers. The third level used software algorithms executed on a computer farm to reconstruct tracks in the central tracking system and required at least one track with transverse momentum $p_{T}>10 \mathrm{GeV}$. The integrated luminosity of the selected sample is $226 \mathrm{pb}^{-1}$ determined with a $6.5 \%$ uncertainty [7].

After full reconstruction, the events were required to have an isolated muon with $p_{T}^{\mu}>12 \mathrm{GeV}$ and a $\tau$ candidate. The muon isolation required less than $4 \mathrm{GeV}$ in the calorimeter in a cone $R \equiv \sqrt{(\Delta \phi)^{2}+(\Delta \eta)^{2}}<0.1$ (where $\phi$ is the azimuthal angle and $\eta$ is the pseudorapidity) around the muon, less than $4 \mathrm{GeV}$ in an annulus $0.1<R<0.4$, and fewer than three tracks (other than the muon) with $p_{T}>0.25 \mathrm{GeV}$ within $R<0.7$.

Most $\tau$ leptons decay to one or three long lived charged particles plus up to three $\pi^{0}$ mesons that can be observed in the detector. The $\tau$ candidates were found by constructing a calorimeter cluster made of all the towers with energy above a preset threshold around a seed tower within $R<0.5$, keeping only clusters with $E_{T}^{\tau}>5 \mathrm{GeV}$ and $E_{T}^{\text {core }}>4 \mathrm{GeV}$, where $E_{T}^{\tau}\left(E_{T}^{\text {core }}\right)$ is the transverse energy with respect to the beam axis within $R<0.5$ $(R<0.3)$, and requiring $r m s_{\tau}<0.25$ (see Table \ caption) and at least one associated track with $p_{T}>1.5 \mathrm{GeV}$ within $R<0.3$. If there was more than one track, the one with highest $p_{T}$ was associated with the $\tau$ candidate. A second track was added if the invariant mass calculated from the tracks was less than $1.1 \mathrm{GeV}$, and a third if the invariant mass was less than $1.7 \mathrm{GeV}$ and the total charge was not \pm 3 . Candidates with total charge zero were discarded. Finally, subclusters were constructed from the cells in the EM section of the calorimeter belonging to the
TABLE I: Event pre-selection cuts

\begin{tabular}{lr}
\hline \hline Selection & applied to the $\tau$-types \\
\hline only one $\mu$ & all \\
$p_{T}^{\mu}>12 \mathrm{GeV}$ & all \\
$\mu$ isolation & all \\
$E_{T}^{\tau}>10(5) \mathrm{GeV}$ & 1 and $3(2)$ \\
$\Sigma p_{T}^{\tau_{t r k}}>7(5) \mathrm{GeV}$ & 1 and $3(2)$ \\
$r m s_{\tau}<0.25^{a}$ & all \\
$\left|\phi_{\mu}-\phi_{\tau}\right|>2.5$ & all \\
$\mathcal{R}_{t r k}^{\tau}>0.7^{b}$ & 1 and 2 \\
\hline \hline
\end{tabular}

${ }^{a} r m s_{\tau}=\sqrt{\sum_{i=1}^{n}\left[\left(\Delta \phi_{i}\right)^{2}+\left(\Delta \eta_{i}\right)^{2}\right] E_{T_{i}} / E_{T}}$, where $i=1, . ., n$ is the index of the calorimeter tower associated with the $\tau$-cluster; $\Delta \eta_{i}$ and $\Delta \phi_{i}$ are the $\eta$ and $\phi$ difference between the center of the $\tau$-cluster and calorimeter tower $i$.

${ }^{b} \mathcal{R}_{\text {trk }}^{\tau}=\left(E^{\tau}-E_{C H}^{t r k}\right) / p_{T}^{t r k}$, where $E_{C H}^{t r k}$ is the energy deposited in a window of $5 \times 5$ towers (each tower of size $\phi \times \eta=0.1 \times 0.1$ ) around the $\tau$-track in the coarse hadronic $(\mathrm{CH})$ section of calorimeter.

$\tau$-cluster. The minimum $E_{T}$ required for an EM subcluster was $800 \mathrm{MeV}$. Three types of $\tau$ candidates were identified according to tracking and calorimetry information: 1) single track with no subclusters in the electromagnetic (EM) section of the calorimeter ( $\pi$-like), 2) single track with EM subclusters ( $\rho$-like), or 3$)$ more than one associated track. No attempt was made to separate hadrons from electrons (which can contribute to both $\tau$-type 1 and $\tau$-type 2 ).

Additional requirements (which depend on the $\tau$-type) imposed on the selected events to enhance the signal-tobackground ratio are shown in Table 1 The background increases rapidly with decreasing $p_{T}^{\mu}$ or decreasing $E_{T}^{\tau}$. It is significantly lower for $\tau$-type 2 than for the other $\tau$ types, so a lower $E_{T}^{\tau}$ cut is warranted for that $\tau$-type. The $\left|\phi_{\mu}-\phi_{\tau}\right|>2.5$ cut takes advantage of the fact that most $Z$ bosons have low $p_{T}$ and thus the decay $\tau$ leptons are back-to-back in $\phi$. The longitudinal shape variable $\mathcal{R}_{\text {trk }}^{\tau}$ (defined in Table @ caption) is used to remove misidentified muons because it has a distribution that peaks at much lower values for muons than for $\tau$ leptons.

The $\tau$ leptons from a $Z$ boson decaying to hadrons + $\nu_{\tau}$ have average visible energy $\left(E^{\tau}\right)$ of the order of 25 $\mathrm{GeV}$ and need to be separated from a very large background of jets. To further reduce the jet background, a neural network (NN) 8] consisting of a single input layer containing several nodes (one for each input variable), a single hidden layer with the same number of nodes, and a single output node was used. A separate NN was trained for each type using a Monte Carlo (MC) sample of single $\tau$ leptons uniformly distributed in $E_{T}$ and $\eta$ and overlayed with a minimum bias event for signal [9], and jets recoiling against non-isolated muons from data for background. The NN input variables were chosen to minimize the dependence on the $\tau$ energy and to exploit the narrow width of the energy deposition in the calorimeter, the low track multiplicity, the low $\tau$ mass, and the fact that $\tau$ leptons from $Z$ boson decays are well isolated. The NN 
input variables were:

1. profile $=\left(E_{T_{1}}+E_{T_{2}}\right) / E_{T}^{\tau}$, where $E_{T_{1}}$ and $E_{T_{2}}$ are the $E_{T}$ of the two most energetic calorimeter towers. Used for all $\tau$-types.

2. caliso $=\left(E_{T}^{\tau}-E_{T}^{\text {core }}\right) / E_{T}^{\text {core }}$. A calorimeter isolation parameter used for all $\tau$-types.

3. $t$ triso $=\Sigma p_{T}^{t r k} / \Sigma p_{T}^{\tau_{t r k}}$, where $p_{T}^{\text {trk }}\left(p_{T}^{\tau_{t r k}}\right)$ is the $p_{T}$ of a track within a $R<0.5$ cone not associated (associated) with the $\tau$ candidate. A track isolation parameter used for all $\tau$-types.

4. $\left(E^{E M_{1}}+E^{E M_{2}}\right) / E^{\tau}$ in a $R<0.5$ cone, where $E^{E M_{1}}$ and $E^{E M_{2}}$ are the energies deposited in the first two layers of the EM calorimeter. A parameter used for $\tau$-type 1 to reject jets with one energetic charged track and soft $\pi^{0}$ mesons.

5. $p_{T}^{\tau_{t r k 1}} / E_{T}^{\tau}$, where $p_{T}^{\tau_{t r k 1}}$ is $p_{T}$ of the highest $p_{T}$ track associated with the $\tau$. Used for $\tau$-type 1 and 3 .

6. $p_{T}^{\tau_{t r k 1}} /\left(E_{T}^{\tau} \cdot\right.$ caliso $)$. A parameter used for $\tau$-type 2 that measures the correlation between track and energy deposition in isolation annulus.

7. $e_{12}=\sqrt{\Sigma p_{T}^{\tau_{t r k}} \cdot E_{T}^{E M}} / E_{T}^{\tau}$, where $E_{T}^{E M}$ is the transverse energy deposited in the EM layers of the calorimeter. Used for $\tau$-types 2 and 3 .

8. $\delta \alpha=\sqrt{(\Delta \phi / \sin \theta)^{2}+(\Delta \eta)^{2}}$, where the differences are between $\Sigma \tau$-tracks and $\Sigma$ EM-clusters. In the small angle approximation the observed $\tau$ mass is given by $e_{12} \cdot E_{T}^{\tau} \cdot \delta \alpha$. Used for $\tau$-types 2 and 3 .

The dominant background is from multijet (QCD) processes, mainly from $b \bar{b}$ events where the muon isolation requirement is met and a jet satisfies the $\tau$ selection criteria. The other sources of background are $W \rightarrow \mu \nu+$ jets and $Z / \gamma^{*} \rightarrow \mu^{+} \mu^{-}$with one of the muons misidentified as a $\tau$ lepton. The $\mathcal{R}_{\text {trk }}^{\tau}>0.7$ cut removed $70 \%$ of the $\mu^{+} \mu^{-}$background while keeping $98 \%$ of the expected $Z / \gamma^{*} \rightarrow \tau^{+} \tau^{-}$events. The number of events that did not satisfy this criterion was used to estimate the background from misidentified muons remaining in the sample after the cut.

The selected 29,021 events were separated into two samples: $\mu$ and $\tau$ of opposite charge sign (OS), and $\mu$ and $\tau$ of same charge sign (SS). The OS sample contains the signal. The SS sample is dominated by background and was used to predict the QCD background distributions in the signal sample. From detailed studies of a sample of data with non-isolated muons, we established that this procedure is sound if one accounts for a small excess of OS over SS events that varies somewhat with the $\tau$-type. The correction factors $\left(f_{i}\right.$, where $i$ denotes the $\tau$-type) were determined to be $1.06 \pm 0.06,1.09 \pm 0.03$, and $1.03 \pm 0.02$, by taking the ratio of OS to SS data in the non-isolated muon sample. There was no observable dependence of $f_{i}$ as function of $E_{T}^{\tau}$, of NN output $(N N)$ values, or of the muon parameters. A possible dependence of $f_{i}$ on the degree of isolation of muons coming from jets was checked by looking at the variation in the $f_{i}$ as function of muon $p_{T}$ relative to the jet axis and varying the muon isolation. No variation was observed within the systematic uncertainties quoted. An overall $3 \%$ systematic uncertainty was added for the extrapolation to the $N N>0.8$ region. These factors do not fully account, however, for the contribution from $W \rightarrow \mu \nu+$ jets, which have a larger excess of OS over SS and different distributions. The additional contribution of this channel to the signal sample is estimated from PYTHIA 9] MC samples. The MC is normalized using the OS and SS data with $p_{T}^{\mu}>20 \mathrm{GeV},\left|\phi_{\mu}-\phi_{\tau}\right|<2.0$, and $0.3<N N<0.8$ (in this region $W \rightarrow \mu \nu$ events dominate over the QCD background). The additional contribution to the background from $W \rightarrow \tau \nu$ events was ignored as it is a small fraction of the uncertainty on the contribution from $W \rightarrow \mu \nu$ events.

Figure 1 shows the $N N$ distributions for each $\tau$-type (and the sum) for the signal sample, the predicted background and the result of adding the predicted signal (from $Z / \gamma^{*} \rightarrow \tau \tau$ MC 9]) to the background. Table II shows the total number of events observed and predicted before and after the final cut $N N>0.8$. Distributions of background subtracted data are in very good agreement with those expected from $Z \rightarrow \tau \tau$ MC. Figure 2 compares the expected $E_{T}^{\tau}$ and $p_{T}^{\mu}$ (adding all $\tau$-types) signal distributions to the predicted background distributions, and to the distributions obtained by subtracting the predicted background from the signal sample distributions.

The total event efficiency $\left(\epsilon_{\mathrm{TOT}}\right)$ summed over $\tau$-types 1,2 , and 3 is $1.52 \%$ for $M_{\tau \tau}$ greater than $60 \mathrm{GeV}$. The total efficiency accounts for all losses due to branching ratios, geometrical acceptance, reconstruction and trigger efficiencies. It is corrected for the small difference between MC and data reconstruction efficiencies. The contributions of the three $\tau$-types to the signal in the final data sample are $13 \%, 58 \%$, and $29 \%$.

The cross section times branching ratio for $Z / \gamma^{*} \rightarrow$ $\tau^{+} \tau^{-}$is given by $N_{\text {signal }} /\left(\epsilon_{\mathrm{TOT}} \cdot \int \mathcal{L} d t\right)$ where $N_{\text {signal }}$ is given by the number of signal events and $\int \mathcal{L} d t$ is the integrated luminosity of the sample studied. $N_{\text {signal }}=$ $865 \pm 55$ (statistical uncertainty only) is the number of OS events of all $\tau$ types after selecting the events with $N N>0.8$, subtracting the estimated background (see Table (II), and subtracting the number of expected events in the sample with $M_{\tau \tau}$ less than $60 \mathrm{GeV}(3.5 \%)$.

The systematic uncertainties on the cross section measurement are listed in Table III The uncertainty $(2.5 \%)$ due to the energy scale was estimated from the change in the acceptance when scaling the energy in MC events by the energy difference between MC and data (as de- 

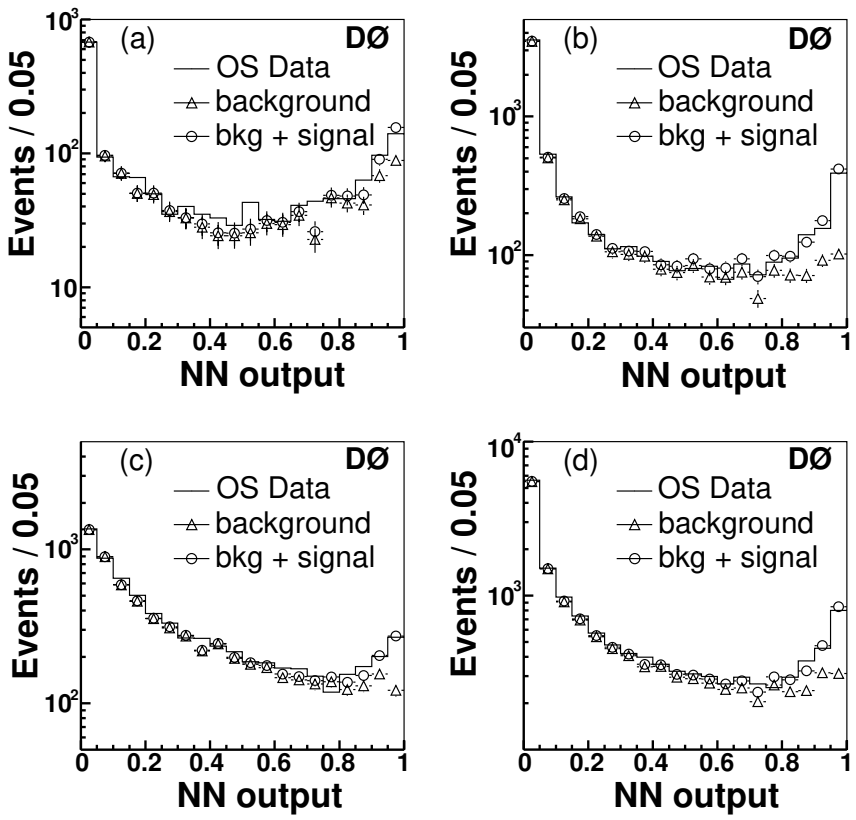

FIG. 1: NN output distributions for: (a) $\tau$-type 1, (b) $\tau$-type 2 , (c) $\tau$-type 3 , and (d) the sum over all the $\tau$-types.

TABLE II: Number of predicted and observed contributions to OS events by $\tau$-type before and after the $N N>0.8$ cut

\begin{tabular}{lcccc}
\hline \hline & $\tau$-type 1 & $\tau$-type 2 & $\tau$-type 3 & Total \\
\hline $\mathrm{QCD}^{a}$ & $1638 \pm 107$ & $6001 \pm 187$ & $6242 \pm 153$ & $13881 \pm 264$ \\
$Z / \gamma^{*} \rightarrow \mu \mu$ & $33 \pm 11$ & $67 \pm 22$ & - & $100 \pm 24$ \\
$W \rightarrow \mu \nu^{b}$ & $42 \pm 41$ & $151 \pm 93$ & $241 \pm 114$ & $434 \pm 153$ \\
$Z / \gamma^{*} \rightarrow \tau \tau^{c}$ & $139 \pm 6$ & $700 \pm 26$ & $335 \pm 14$ & $1174 \pm 43$ \\
\hline Sum & $1852 \pm 117$ & $7019 \pm 214$ & $6818 \pm 189$ & $15589 \pm 309$ \\
OS events & 1880 & 6971 & 7060 & 15911 \\
\hline \hline \multicolumn{5}{c}{$N N>0.8$} \\
QCD & $196 \pm 23$ & $280 \pm 24$ & $508 \pm 32$ & $984 \pm 46$ \\
$Z / \gamma^{*} \rightarrow \mu \mu$ & $30 \pm 10$ & $40 \pm 13$ & - & $70 \pm 16$ \\
$W \rightarrow \mu \nu$ & $3 \pm 5$ & $17 \pm 11$ & $38 \pm 16$ & $58 \pm 20$ \\
$Z / \gamma^{*} \rightarrow \tau \tau$ & $121 \pm 6$ & $532 \pm 21$ & $261 \pm 11$ & $914 \pm 24$ \\
\hline Sum & $350 \pm 26$ & $869 \pm 36$ & $807 \pm 37$ & $2026 \pm 57$ \\
OS events & 355 & 820 & 833 & 2008 \\
\hline \hline
\end{tabular}

${ }^{a}$ The QCD background is estimated by multiplying the number of SS events by $f_{i}$ (described in the text).

${ }^{b}$ The expected contribution is the number of events that must be added after subtracting the corrected number of SS events from OS events.

${ }^{c}$ The predicted number of $Z / \gamma^{*} \rightarrow \tau^{+} \tau^{-}$events is based on a theoretical cross section of $257 \pm 9 \mathrm{pb}$ for $M_{\tau \tau}>60 \mathrm{GeV}[10]$ plus $3.5 \%$ predicted from $\mathrm{MC}$ for the number of events expected with $M_{\tau \tau}<60 \mathrm{GeV}$.

termined by the $p_{T}$ imbalance in photon + jet events). The systematic uncertainty due to the NN performance $(2.6 \%)$ was estimated by generating ensembles of Monte Carlo events in which the number of events in each bin of distributions of NN input variables was allowed to fluctuate by the uncertainties in the difference between MC distributions and the background-subtracted data distribu-
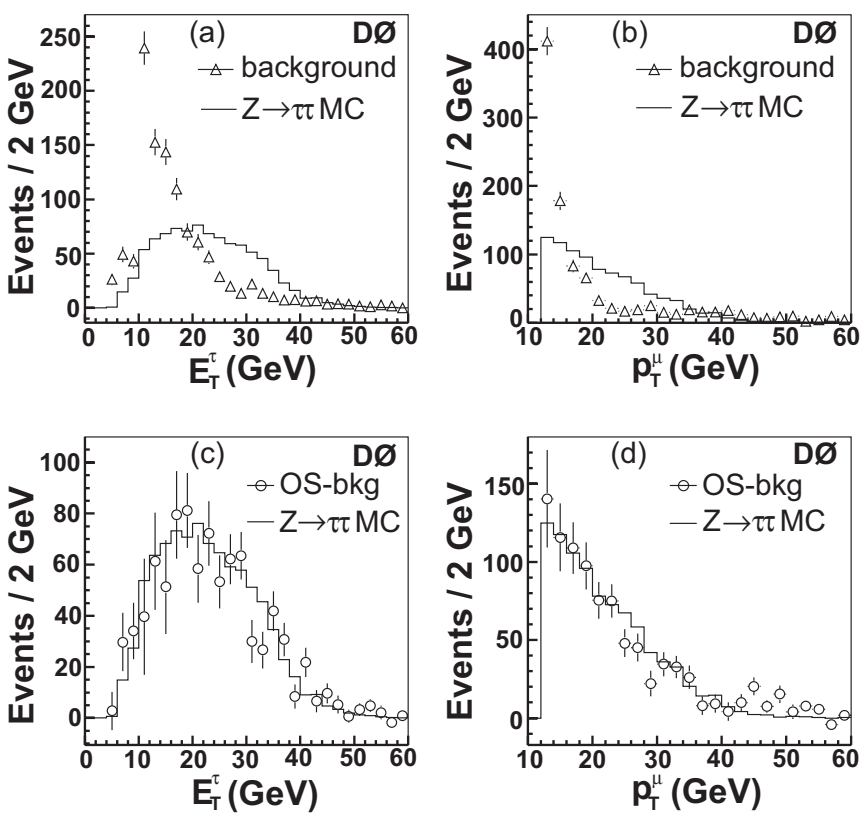

FIG. 2: $\quad E_{T}^{\tau}[(\mathrm{a}),(\mathrm{c})]$ and $p_{T}^{\mu}[(\mathrm{b}),(\mathrm{d})]$ distributions after $N N>0.8$ cut: (a), (b) estimated background (open triangles) and predicted $Z \rightarrow \tau \tau$ signal (histogram); (c), (d) background subtracted data (open circles) and predicted $Z \rightarrow \tau \tau$ signal.

TABLE III: Systematic uncertainties on $\sigma \cdot \operatorname{Br}\left(Z / \gamma^{*} \rightarrow \tau^{+} \tau^{-}\right)$

\begin{tabular}{lr}
\hline \hline Energy Scale & $2.5 \%$ \\
$\mathrm{NN}$ & $2.6 \%$ \\
$\mathrm{QCD}$ background & $3.5 \%$ \\
$Z / \gamma^{*} \rightarrow \mu \mu$ background & $2 \%$ \\
$W \rightarrow \mu \nu$ background & $2.3 \%$ \\
$Z / \gamma^{*} \rightarrow \tau \tau \mathrm{MC}$ & $1.5 \%$ \\
$\mathrm{PDF}^{a}$ & $1.7 \%$ \\
$\epsilon_{\mathrm{data}} / \epsilon_{M C}{ }^{b}$ & $2.1 \%$ \\
Trigger & $3.5 \%$ \\
\hline Total & $7.5 \%$ \\
\hline \hline
\end{tabular}

${ }^{a}$ Efficiency uncertainty due to uncertainty in parton distribution function (PDF).

${ }^{b} \epsilon_{\mathrm{d} a t a} / \epsilon_{M C}$ is the ratio of data to MC reconstruction efficiency.

tions. The distributions of $\mathrm{NN}$ input variables are in good agreement with those predicted adding $Z / \gamma^{*} \rightarrow \tau^{+} \tau^{-}$ $\mathrm{MC}$ and the estimated background; two are shown in Fig. 3

The QCD systematic uncertainty $(3.5 \%)$ is due to the uncertainty in determining $f_{i}$. The uncertainty in the $Z / \gamma^{*} \rightarrow \mu^{+} \mu^{-}$and $W \rightarrow \mu \nu$ backgrounds $(2.0 \%$ and $2.3 \%$ ) come from the statistical uncertainty in determining their contribution, while the $Z / \gamma^{*} \rightarrow \tau^{+} \tau^{-}$MC systematic uncertainty reflects limited signal MC statistics. The $\epsilon_{\mathrm{data}} / \epsilon_{M C}$ is dominated by the uncertainty in estimating the difference in $\tau$-type 3 tracking efficiency between $\mathrm{MC}$ and data using the the ratio of two- to three- 

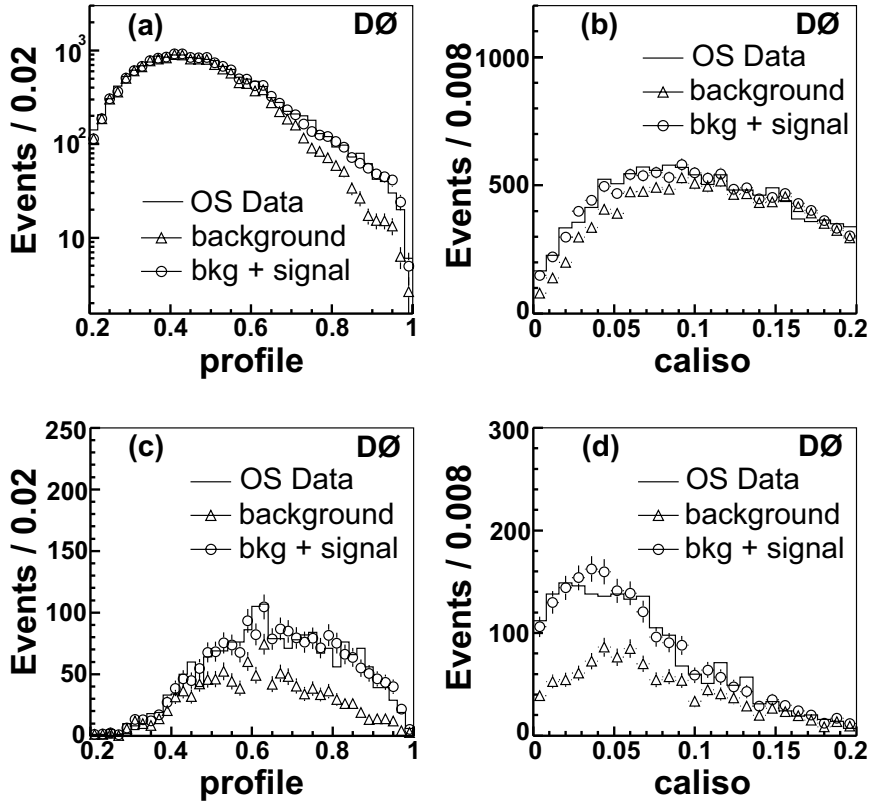

FIG. 3: Distributions for OS data, background and background plus signal of two NN input variables before [(a), (b)] and after $[(\mathrm{c}),(\mathrm{d})] N N>0.8$ cut: (a),(c) profile; (b),(d) caliso.

prong events between background subtracted data and $Z / \gamma^{*} \rightarrow \tau^{+} \tau^{-} \mathrm{MC}$ in $\tau$-type 3 candidates. The uncertainty from differences in subcluster reconstruction, single isolated track reconstruction and muon isolation add up to about $1 \%$.

The trigger efficiencies were estimated using $Z \rightarrow$ $\mu^{+} \mu^{-}$data, the systematic uncertainty comes from the statistical uncertainty in that data; the uncertainties include dependencies on $\eta$ and $\phi$. Systematic uncertainties from all other sources are less than $1 \%$. Thus we obtain

$$
\sigma \cdot \operatorname{Br}\left(Z / \gamma^{*} \rightarrow \tau \tau\right)=252 \pm 16(\text { stat }) \pm 19(\text { sys }) \mathrm{pb}
$$

for $M_{\tau \tau}$ greater than $60 \mathrm{GeV}$. The quoted statistical uncertainty is the uncertainty from OS and SS statistics (excluding the uncertainties on the correction factors). This yields, after removing the $\gamma^{*}$ contribution,

$\sigma \cdot \operatorname{Br}(Z \rightarrow \tau \tau)=237 \pm 15($ stat $) \pm 18($ sys $) \pm 15($ lum $) \mathrm{pb}$

in good agreement with the NNLO standard model prediction of $242 \pm 9 \mathrm{pb}$ 10.

We thank the staffs at Fermilab and collaborating institutions, and acknowledge support from the Department of Energy and National Science Foundation (USA), Commissariat à l'Energie Atomique and CNRS/Institut National de Physique Nucléaire et de Physique des Particules (France), Ministry of Education and Science, Agency for Atomic Energy and RF President Grants Program (Russia), CAPES, CNPq, FAPERJ, FAPESP and
FUNDUNESP (Brazil), Departments of Atomic Energy and Science and Technology (India), Colciencias (Colombia), CONACyT (Mexico), KRF (Korea), CONICET and UBACyT (Argentina), The Foundation for Fundamental Research on Matter (The Netherlands), PPARC (United Kingdom), Ministry of Education (Czech Republic), Canada Research Chairs Program, CFI, Natural Sciences and Engineering Research Council and WestGrid Project (Canada), BMBF and DFG (Germany), A.P. Sloan Foundation, Research Corporation, Texas Advanced Research Program, and the Alexander von Humboldt Foundation.

[*] Visitor from University of Zurich, Zurich, Switzerland.

[1] UA1 Collaboration, C. Albajar et al., Phys. Lett. B 253, 503 (1991). UA2 Collaboration, J. Alitti et al., Phys. Lett. B 276, 365 (1992).

[2] CDF Collaboration, T. Afforder et al., Phys. Rev. Lett. 84, 845 (2000), F. Abe et al., Phys. Rev. D59, 052002 (1999). CDF Collaboration, D.Acosta et al. hep-ex/0406078 (2004).

[3] DØ Collaboration, S. Abachi et al., Phys. Rev. Lett. 75, 1456 (1995), B. Abbot et al., Phys. Rev. D 60, 053003 (1999)

[4] OPAL Collaboration, G. Abbiendi et al., Eur. Phys. J. C 19, 587 (2001). DELPHI Collaboration, P. Abreu et al., Eur. Phys. J. C 16, 371 (2000). L3 Collaboration, M. Acciarri et al., Eur. Phys. J. C 16, 1 (2000). ALEPH Collaboration, R. Barate et al., Eur. Phys. J. C 14, 1 (2000).

[5] DØ Collaboration, S. Abachi et al. Nucl. Instrum. Methods Phys. Res., Sect. A 338, 185 (1994), contains a detailed description of the calorimeter. DØ Collaboration, V. Abazov et al. in preparation for submission to Nucl. Instrum. Methods Phys. Res. A, T. LeCompte and H.T Diehl, Ann. Rev. Nuc. Part. Sci. 5071 (2000).

[6] DØ Collaboration, V. Abazov et al., Phys. Rev. Lett. 93, 141801 (2004).

[7] T. Edwards et al., FERMILAB-TM-2278-E.

[8] C. Peterson, T. Rögnvaldsson, and L. Lönnblad, Comput. Phys. Commun. 81, 185 (1994).

[9] We used the tau decay library TAUOLA: S. Jadach et al., Comput.Phys.Commun. 76 (1993) 361 for $\tau$ decays, the PYTHIA program to generate minimum bias events: T. Sjöstrand et al., Comput. Phys. Commun. 135, 238 (2001), and the GEANT program for detector simulation: R. Brun et al. CERN Program Library Writeup W5013 (1993).

[10] The theoretical cross section was determined using the NNLO calculations from R. Hamberg, W.L. van Neerven, and T. Matsuura, Nucl. Phys. B359, 343 (1991), updated to the most recent parton distribution function (CTEQ6) from J. Pumplin et al., JHEP 0207012 (2002) and D. Stump et al., JHEP 0310046 (2003) . 